Reprinted from The Journal of the Acoustical Society of America, Vol. 27, No. 5, 913-921, September, 1955

Copyright, 1955, by the Acoustical Society of America.

Printed in U. S. A.

\title{
Production of Accelerated Cavitation Damage by an Acoustic Field in a Cylindrical Cavity*
}

\author{
A. T. ELLIS \\ California Institute of Technology, Pasadena, California
}

(Received April 8, 1955)

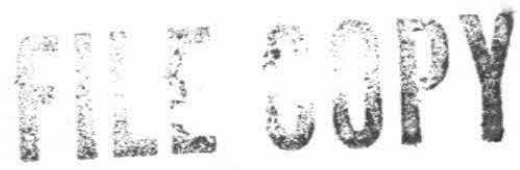

\begin{abstract}
A convenient and economical method for the production of cavitation damage is described. Cavitation is generated in the region of the maximum pressure amplitude of an acoustic field in a resonant cylindrical container. The specimen surface to be damaged is a stationary boundary in the system and is therefore not subject to accelerations such as occur in conventional magnetostriction devices for cavitation damage study. The absence of these accelerations eliminates any possibility of premature material removal from cavitation weakened surfaces. Mathematical analysis of the dynamics of cavitation should also be facilitated. It is shown that simple assumptions lead to an acoustical theory agreeing with experiment to within the accuracy required. Examples are given of the application of this technique to the study of both easily damaged and highly resistant materials. Relatively uniform damage is achieved which is particularly suitable for $\mathrm{x}$-ray diffraction study of the surface. Alternatively, perturbation of the acoustic field to permit concentrated damage is obtained by forming the specimen surface as the end of a small cylinder. Photomicrographic studies of damage to both monocrystalline and polycrystalline materials have been initiated with the aid of this apparatus. Cavitation experiments have been done in water with an air atmosphere and in toluene with a helium atmosphere. These experiments show that severe damage can result even if a chemically inert environment is employed.
\end{abstract}

\section{INTRODUCTION}

$\mathrm{C}$ AVITATION is the occurrence of cavities of vapor or vapor and gas phase in a region of liquid phase. These cavities, or bubbles, may be formed in various ways, but the essential requirement for cavitation is the existence of a region of liquid in which the absolute pressure is less than the vapor pressure of the liquid. If the liquid is relatively free of gaseous or solid particles which act as bubble nuclei, then large tensions may be required for bubble formation. ${ }^{1}$

Pressure variation by flow over submerged bodies in the generating process usually considered when cavitation is mentioned. This fact follows from the practical importance of damage to hydraulic machinery such as ship propellers, pumps, and turbines resulting from the collapse of cavities produced in this manner. More recently, the increased use of ultrasonic devices has made cavitation damage an important consideration in the design of high power transducers for use in liquids.

The mechanism of cavitation and the resulting damage to surfaces is still not completely understood after more than a half-century of investigation. The extensive work which has been done indicates that a great number of variables are important and that there are many interactions between them. It has become clear, however, that large mechanical forces are present in the damage process. ${ }^{2}$ Physical properties of both solid and liquid are therefore of prime importance, and

\footnotetext{
* This work was supported by the Ofice of Naval Research, under contract N6onr-24420 (NR 062-059). Reproduction in whole or in part is permitted for any purpose of the U. S. Government.

${ }^{1}$ W. J. Galloway, "An experimental study of acoustically induced cavitation," University of California at Los Angeles, Department of Physics, Tech. Rept. No. 7 (1953).

${ }^{2}$ M. S. Plesset and A. T. Ellis, "On the mechanism of cavitation damage," Trans. Am. Soc. Mech. Engrs. (to be published).
}

cooperation between hydrodynamicists and metallurgists is imperative if more than a mere cataloging of material resistance to cavitation is to be attained.

The equipment and techniques described here were developed as a result of search for a simple method of obtaining cavitation damage quickly and with a minimum of factors involved in the physical situation. It was also felt highly desirable to reduce, if possible, the complications in both electrical and mechanical design inherent in some existing cavitation damage testing machines. A particular requirement of the present program was to obtain an evenly damaged surface for the purpose of examination by $x$-ray diffraction. If the damage were uneven, then results would be influenced by the position of the $x$-ray beam which usually has small cross section. A region of bubble formation where space gradients in the pressure field are kept small is therefore required. Furthermore, it has been found that pressure gradients are important factors in determining the geometry of bubble collapse. ${ }^{3}$ Alternatively, it is sometimes desirable to obtain small regions of intense field in order to quickly damage extremely resistant materials without large energy dissipation which would introduce cooling problems. Provision for the latter type of damage was therefore made.

\section{DESCRIPTION OF METHOD AND APPARATUS}

It has long been recognized that cavitation produced by pressure variation at a relatively high frequency will cause damage much more rapidly than cavitation due to flow. When working with the cavitation resistant materials which are of most interest, it is therefore more

\footnotetext{
${ }^{3}$ A. T. Ellis, "Observations on cavitation bubble collapse," California Institute of Technology, Hydrodynamics Laboratory, Rept. No. 21-12 (1952).
} 


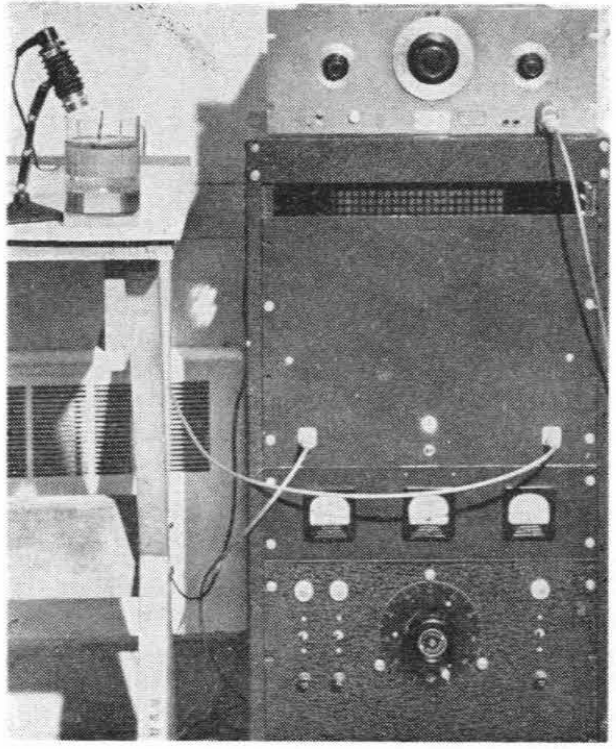

FIG. 1. Complete apparatus including oscillator, amplifier, and beaker.

practical to use the former method. The usual technique for accomplishing this has been the rapid normal motion of the fluid bounding surface which is to be damaged. This motion is often obtained by employing the magnetostrictive effect in a nickel rod to the end of which has been attached the specimen surface. ${ }^{4,5}$ The quantities measured are the displacement of the surface and its frequency. Weight loss of the specimen is obtained as a function of exposure time. The technique here described differs in that the surface to be damaged is practically stationary, and the acoustic field causing cavitation is generated not by motion of the specimen but by a separate transducer. Inertial forces in the sample are thus kept at a minimum. This situation is desirable because small regions of reduced strength are produced in the specimen as cavitation damage progresses and these small regions might be torn out under mechanical acceleration. It is anticipated that analytic consideration of the bubble behavior will be made with the aid of high-speed photographic techniques developed by the author. ${ }^{3}$ From this standpoint it would also seem advantageous to have a stationary boundary and no gross liquid velocity or acceleration.

Figure 1 shows the complete apparatus set up and in operation. It consists of a conventional audiofrequency oscillator and amplifier together with a beaker in which the cavitation is produced. Figure 2 is a close-up view of the beaker and cavitation bubble cloud. It will be noted that the beaker has a flat face to permit photographs of the bubble cloud. This flat

${ }^{4}$ B. G. Rightmire, "A study of the damage producing mechanism in hydraulic cavitation," $\mathrm{Ph}$.D. thesis, Massachusetts Institute of Technology (1941).

${ }^{5}$ M. Kornfeld and L. Suvarov, J. Appl. Phys. 15, 495-506 (1944). did not noticeably affect its operation. Experimental checks with the theory were made both with this beaker $(800 \mathrm{ml}$ Pyrex) and a smaller one $(600 \mathrm{ml}$ Pyrex). The latter did not have a flat and preserved complete circular symmetry. Figure 3 shows the components, including the barium titanate ring transducer, the stainless steel bottom plate, the specimens to be tested, the closed cell rubber covering for the outer surface of the ring, and the beaker itself.

The barium titanate ring, shown in Fig. 4, is forced to expand and contract by a sinusoidal voltage of up to 200 volts applied to a conductive coating on its inner and outer surfaces. Normal operating frequency is 18 kilocycles for the larger beaker and ring, and 24 kilocycles for the smaller one. The presence of the closed cell rubber over the outer surface of the ring permits better coupling to the normal modes of the system. The specimen is mounted flush in the bottom plate to minimize edge effects except when a concentrated field is desired. In the latter case the specimen consists of a 0.07 inch diameter cylinder projecting 0.07 inch above the bottom plate. A standing wave pattern is set up in such a way that there is large pressure amplitude resulting in cavitation at the surface of the specimen.

The theory of acoustic waves in cylinders has been well developed, principally through a desire to use them as a means of measuring the acoustic impedance of various media. ${ }^{6}$ It has been shown that the general problem which considers arbitrary excitation as well as longitudinal and shear waves in the cylinder walls is quite complex. ${ }^{7}$ The purpose of the present analysis is merely to show that relatively simple assumptions are sufficient to provide results agreeing with experiment within the accuracy required in this application.

In operation, the height of the ring is adjusted to the point where essentially only one mode is excited. The

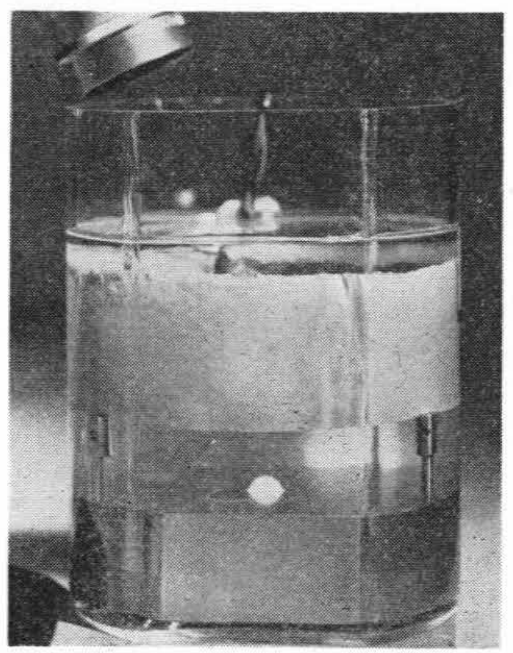

FIG. 2. Beaker in operation showing cavitation cloud.

${ }^{6}$ Fay, Brown, and Fortier, J. Acoust. Soc. Am. 19, 850-856 (1947).

${ }^{7}$ W. J. Jacobi, J. Acoust. Soc. Am. 21, 120-127 (1949). 
geometry of the exciting ring is neglected; this neglect is permissible if the width of the ring is not too great.

In the acoustic approximation and with the neglect of viscosity and streaming effects, one may express the sound field by a velocity potential $\varphi$ from which the particle velocity $V$ and the time dependent portion of the pressure $P$ may be found from the relations

$$
V=-\nabla \varphi, \quad P=\rho_{w} \frac{\partial \varphi}{\partial t}
$$

where $\rho_{w}$ is the density of the liquid, and $\varphi$ satisfies the wave equation

$$
\Delta \varphi-\frac{1}{c^{2}} \frac{\partial^{2} \varphi}{\partial t^{2}}=0 \text {. }
$$

One looks for a characteristic solution of the form $\varphi=R(r) Q(z) e^{i t \omega}$ where $c$ is the sound velocity in the liquid, $\omega / 2 \pi$ is the exciting frequency, $r$ is the radial distance from the axis of cylindrical symmetry, and $z$ is the distance measured along this axis (see Fig. 4). The origin of $z$ is at the water surface and the positive direction of $z$ is upward.

The boundary conditions may be described as follows. The massive steel plate at the bottom of the container is assumed to move as a free rigid mass. Under this assumption, Newton's second law of motion gives a condition on the velocity potential at the interface of the liquid and the steel plate:

or

$$
\rho_{w} \frac{\partial \varphi}{\partial t}-\frac{d}{d l}\left(\frac{\partial \varphi}{\partial z}\right)\left(\rho_{s} d\right)=0, \text { at } z=-l ;
$$

$$
\varphi-\frac{\partial \varphi}{\partial z}\left(\frac{\rho_{s} d}{\rho_{w}}\right)=0, \quad \text { at } \quad z=-l ;
$$

where $\rho_{s}$ is the density of the steel plate and $d$ is its thickness. The boundary condition at the beaker wall is determined under the assumption that this wall is

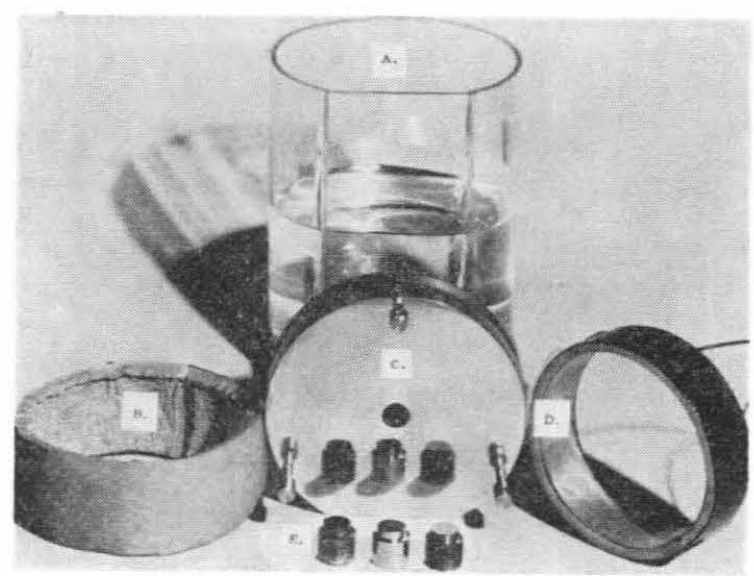

Fig. 3. Disassembled view of components.

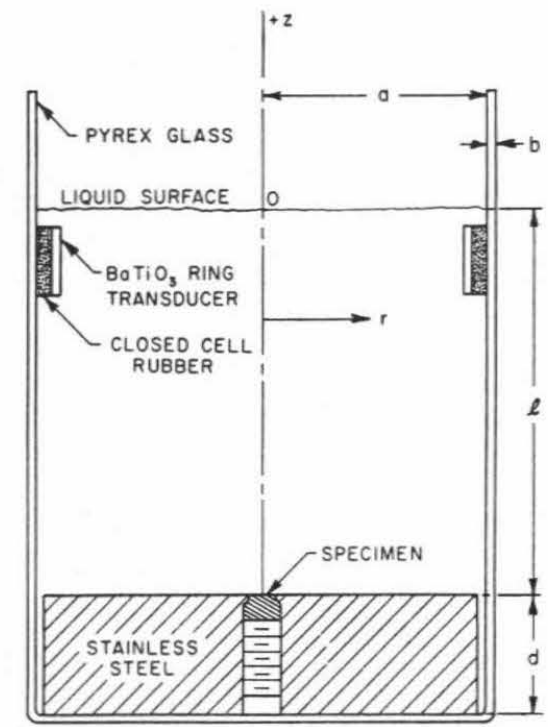

FIG. 4. Diagram of cylindrical beaker and coordinate system.

under simple hoop tension. The force per unit length of the cylindrical wall is $E b d a / a$ where $E$ is the Young's modulus of the glass (Pyrex), $b$ is its thickness, and $a$ is its unstrained radius. Then the force equation at the wall, $r=a$, leads to the boundary condition

$$
\varphi-\left(\frac{E}{a^{2} \omega^{2}}-\rho_{g}\right) \frac{b}{\rho_{w}} \frac{\partial \varphi}{\partial r}=0, \text { at } \quad r=a ;
$$

where $\rho_{g}$ is the density of the glass. There is finally the boundary condition at the free surface of the water:

$$
\varphi=0, \text { at } z=0 .
$$

The characteristic solutions which satisfy these boundary conditions are

$$
\varphi_{m n}=A_{m n} \sin \beta_{m} z J_{0}\left[\left(\frac{\omega_{m n}{ }^{2}}{c^{2}}-\beta_{m}^{2}\right)^{\frac{1}{2}} r\right]
$$

where $J_{0}$ is the Bessel function of zero order and $\beta_{m}$ is a solution of the equation

$$
\frac{\tan \beta l}{\beta}=-\frac{\rho_{s} d}{\rho_{w}} .
$$

The frequency ${ }_{m n}$ is given by

$$
f_{m n}=\frac{c}{2 \pi}\left[\left(\frac{\alpha_{m n}}{a}\right)^{2}+\beta_{m^{2}}\right]^{\frac{1}{2}}
$$

where the constant $\alpha_{m n}$ is determined from the solution of the equation

$$
\frac{J_{0}(\alpha)}{\alpha J_{1}(\alpha)}=-\frac{b}{a}\left\{\frac{\rho_{g}}{\rho_{w}}-\frac{E}{\rho_{w} c^{2}\left[\alpha^{2}+a^{2} \beta_{m}^{2}\right]}\right\} .
$$




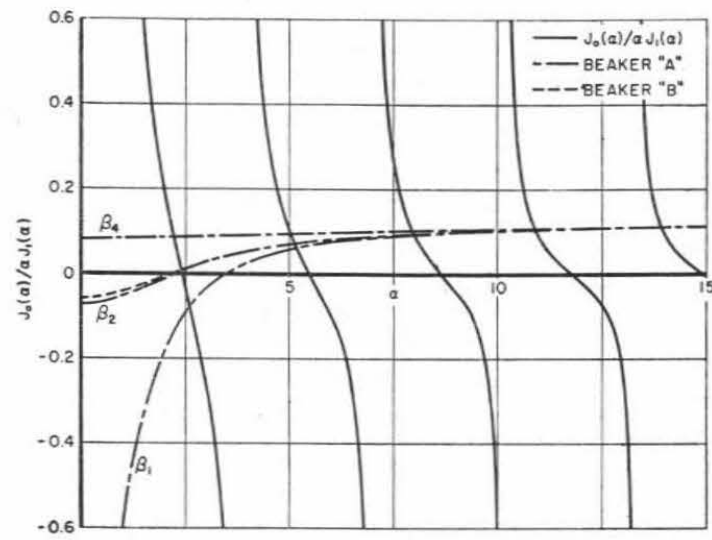

FIG. 5. Graph for the determination of $\alpha_{m n}$.

Figure 5 illustrates a graphical method of solving Eq. (8) for the $\alpha_{m n}$. The left side of this equation is a universal curve which is plotted as a solid line, while the right side, which contains the parameters of the apparatus, is plotted as a dashed curve for each value of $\beta_{m}$ which satisfies $\mathrm{Eq}$. (6). The intersections of the solid and dashed lines give values of $\alpha_{m n}$ which are solutions of Eq. (8). These values are then used in Eq. (7) to obtain the characteristic frequencies. A series of these have been computed and have been compared with measured resonant frequencies for two sets of rings and beakers of different dimensions. These are tabulated in Table I.

Figure 6(a) shows the axial pressure distribution on the left and the radial pressure distribution at different depths on the right for the 2, 1 mode at 18.6 kilocycles. The upper trace (near the liquid surface) is extended out to the beaker wall to show that the pressure variation there is nearly zero at this frequency. From Eq. (3) one finds that $\varphi=0$ at 18.1 kilocycles, which is a good check. On the other hand, for the 1, 2 mode at 27.2 kilocycles shown in Fig. 6(b) the boundary is not free,
TABLE I.

Numerical values of constants

Density of water,

Density of bottom plate,

Density of Pyrex glass,

Young's modulus for Pyrex, $\quad E=6.76 \times 10^{11}$ dynes $/ \mathrm{cm}^{2}$

Velocity of sound in water, $\quad c=1.52 \times 10^{5} \mathrm{~cm} / \mathrm{sec}$

Linear dimensions of apparatus

Beaker A $(800 \mathrm{cc})$ :

$a=4.83 \mathrm{~cm} ; \quad b=0.254 \mathrm{~cm} ; \quad d=2.54 \mathrm{~cm} ; \quad l=8.13 \mathrm{~cm}$ Beaker B $(600 \mathrm{cc})$ :

$a=3.94 \mathrm{~cm} ; b=0.203 \mathrm{~cm} ; \quad d=3.18 \mathrm{~cm} ; \quad l=6.35 \mathrm{~cm}$

Comparison of calculated and observed characteristic frequencies

$\begin{array}{llcc} & f_{\mathrm{mn}} & \begin{array}{c}\text { Calculated } \\ \text { values }\end{array} & \begin{array}{c}\text { Measured } \\ \text { values }\end{array} \\ \text { Beaker A } & f_{11} & 14.0 \mathrm{kc} & 13.6 \mathrm{kc} \\ \text { Beaker A } & f_{21} & 18.6 \mathrm{kc}^{\mathrm{a}} & 18.6 \mathrm{kc} \\ \text { Beaker A } & f_{31} & 26.7 \mathrm{kc} & 26.6 \mathrm{kc} \\ \text { Beaker A } & f_{41} & 34.6 \mathrm{kc} & 33.9 \mathrm{kc} \\ \text { Beaker A } & f_{12} & 26.5 \mathrm{kc} & 27.2 \mathrm{kc} \\ \text { Beaker B } & f_{21} & 23.3 \mathrm{kc}^{\mathrm{s}} & 23.8 \mathrm{kc} \\ \text { Beaker B } & f_{22} & 36.4 \mathrm{kc} & 33.4 \mathrm{kc}\end{array}$

s Normal operating frequencv.

in agreement with theory. Equation (3) also shows that the boundary is more nearly free if $b / \rho_{w}$ is small, corresponding to a thin beaker wall or a dense liquid.

The pressure distributions shown are actual photographs of an oscilloscope screen. The oscilloscope tube plates for vertical deflection were connected to a small pressure probe (a one-eight inch barium titanate cylinder) with its associated amplifier. The probe was mounted on a traversing mechanism coupled to linear variable resistances. In this manner the horizontal trace deflection was made to correspond to either radial (horizontal) or axial (vertical) position of the probs. The photographs show that there is essentially only one mode present, and the distributions conform to

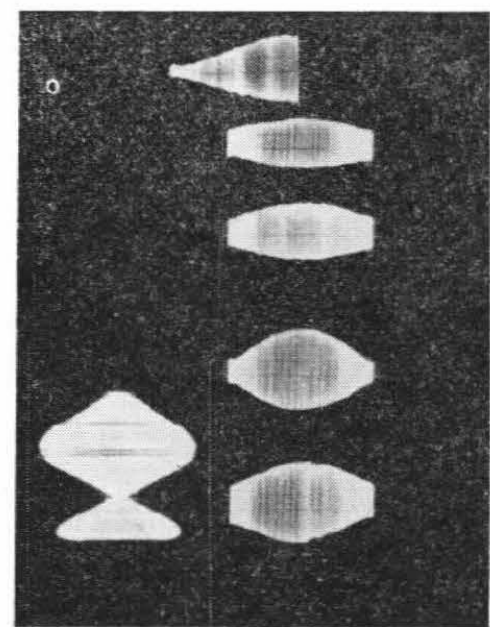

(a)

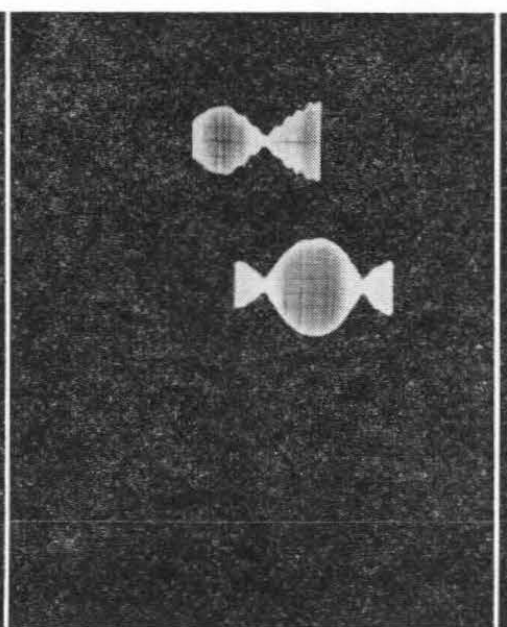

(b)

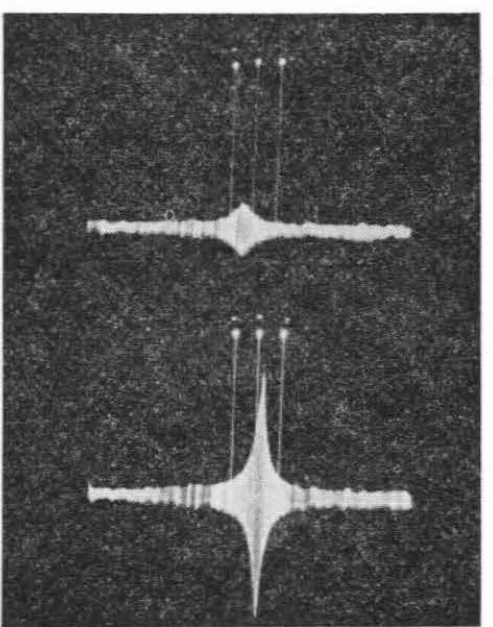

(c)

FIG. 6. Experimental pressure distributions. 
theory. There are, of course, other positions of the ring transducer for which more than one mode is excited. The absolute value of the pressure is known only approximately because of lack of calibration facilities. An estimated value based on calibration by shocktube technique places the peak pressure of the exciting field at from four to five atmospheres for normal damage tests.

As stated previously, when concentrated damage is desired the specimen is formed as a small cylinder projecting upwards from the bottom plate. The damage is greatly increased on the end of this cylinder, enabling one to study extremely resistant materials. A qualitative explanation is that the gradient of the acoustic field near the projection becomes large in a manner somewhat analogous to that of an electrostatic field near a projecting conducting surface. ${ }^{8}$ A quantitative explanation should not be sought on this basis, however, since the presence of the bubbles themselves will greatly modify the field. ${ }^{9}$

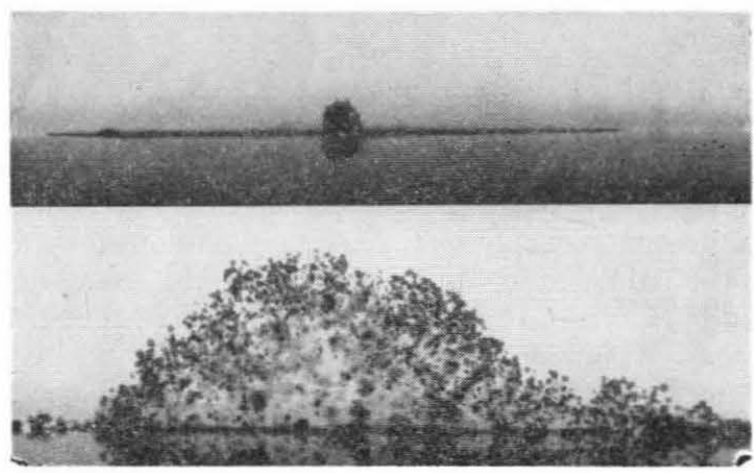

FIG. 7. The bubble cloud at two different points of the pressure cycle.

Figure 6(c) illustrates the marked effect of gas content in the liquid upon operation of the system. The ordinate corresponds to pressure as recorded by the probe in a fixed position near the specimen, while the abscissa is the output of a meter which is a linear measure of frequency. The height and the sharpness of the curve increases as the energy dissipation in the system decreases. The input voltage to the transducer is kept constant, and the only difference between the sharp and broad curves is in the air content of the water used. This illustrates the need for use of degassed liquids in damage tests. Ordinarily, distilled water is used which has been boiled at reduced pressure and room temperature for about one-half hour while subjected to a strong sound field. This procedure results in a much more stable cavitation bubble cloud. If tap water is used without deaeration, the cloud wanders over the specimen and there are frequent bursts of bubbles and much

\footnotetext{
${ }^{8}$ Lord Rayleigh, Theory of Sound (Dover Publications, 1945), Vol. II, p. 293.

'E. L. Carstensen and L. L. Foldy, J. Acoust. Soc. Am. 19, 481591 (1947).
}

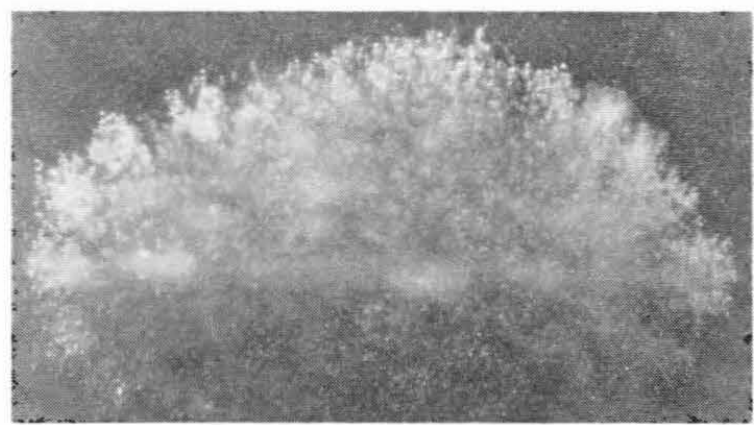

FIG. 8. Bubble cloud with top lighting.

streaming at other points in the liquid. Excessive air content also results in heating of the liquid due to extra energy loss. ${ }^{9,10}$ When deaerated liquid is used, the electrical power input during a test is about 30 watts for the large bubble cloud, and about 6 watts for the small one. The electrical input impedance to the ring is about $600 \mathrm{ohms}$.

\section{EXPERIMENTAL OBSERVATIONS}

Figure 7 shows the larger type of bubble cloud which produces more uniform damage. Two points on the pressure cycle are shown. It will be observed that in the upper photograph, corresponding to higher pressure, the bubbles are nearly collapsed with the exception of a region near the center. Presumably some permanent gas has collected there. These photographs are at a

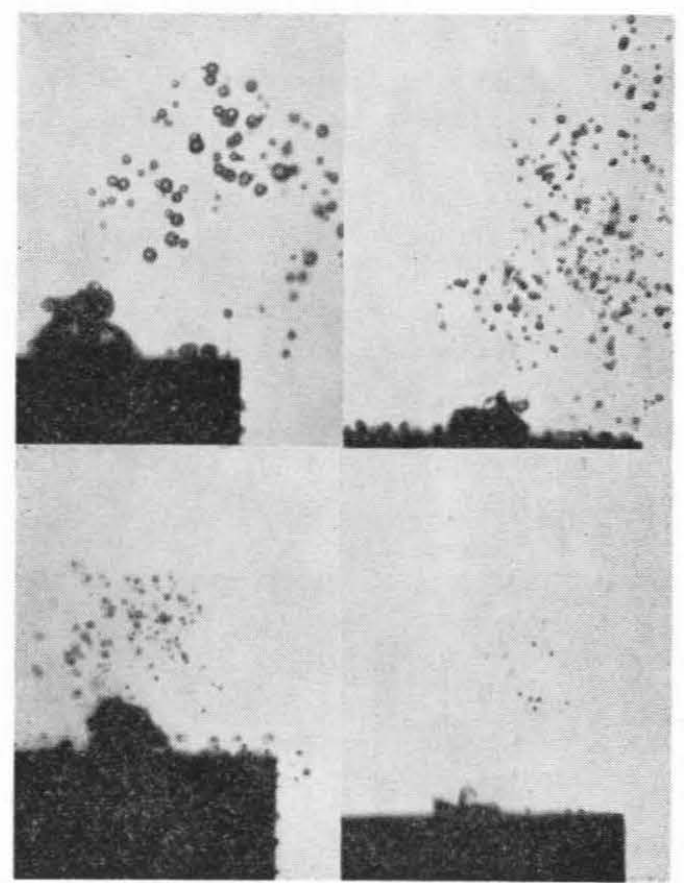

Frg. 9. Bubble cloud over 0.035 inch radius cylinder at different points on the pressure cycle.

${ }^{10}$ Donald T. Laird and Paul M. Kendig, J. Acoust. Soc. Am. 24, 29-32 (1952). 


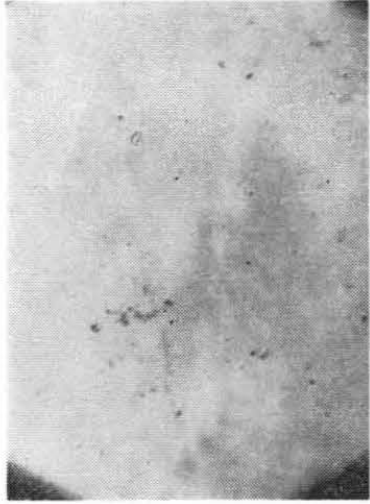

(a)

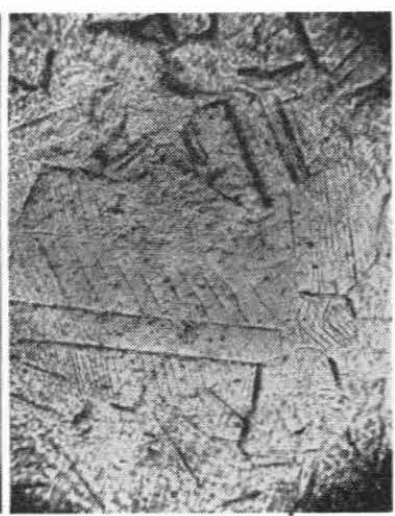

(b)
FIG. 10. Photomicrograph of stainless steel specimen-magnifification $560 \times$ : (a) before exposure to cavitation, (b) after 5 min exposure to cavitation.

magnification of $17 \times$, and are silhouettes. A similar view with top lighting is shown in Fig. 8. A xenon spark gap having a duration of the order of one-half microsecond was used for illumination in both cases.

Figure 9 shows four photographs of the concentrated damage type of cloud on the end of the 0.035 inch radius cylinder. The magnification in this case is $50 \mathrm{X}$.

Although the boundary of the bubble cloud does not appear as well defined as in the case of the larger cloud, a long exposure photograph shows a definite average boundary shaped much like a cosine distribution. Some streaming was observed, however, while these short exposure pictures were being taken. This streaming may account for the boundary irregularity observed here. The magnification is approximately $50 \times$, and it is estimated that bubble radii are measurable down to about $10^{-4}$ inch.

An example of surface photomicrography is illustrated in Fig. 10. The stainless steel clearly shows many slip lines which can only be the result of large mechanical forces. This type of damage is typical of that produced by the larger bubble cloud. Figure 11 shows the uniformity of damage that may be expected. After one-half hour exposure a dark area on the brass specimen indicates the beginning of nonuniform attack. The surface damage at this stage is much more severe than is necessary

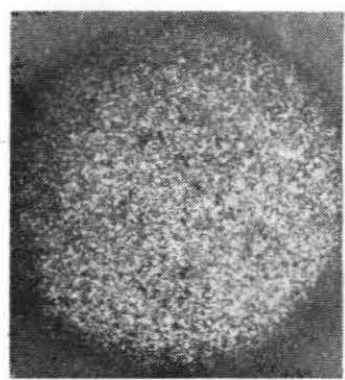

Before damage

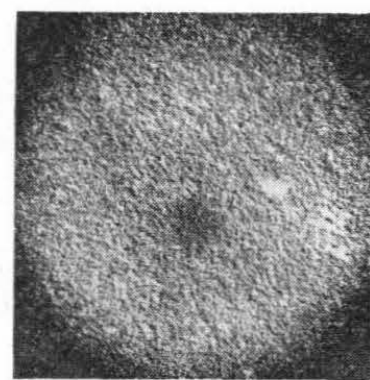

After 30-min exposure
Fig. 11. Damage to fine-grained brass by cavitation of the uniform type.

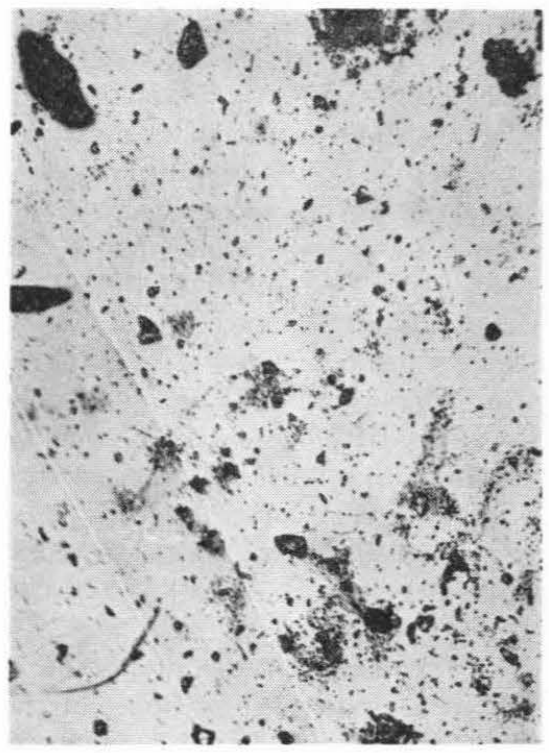

FIG. 12. Annealed surface of a zinc monocrystal cleaved along a basal plane - undamaged $250 \times$.

for $x$-ray examination. Figures 12 and 13 show the striking plastic deformation of a zinc monocrystal by the large cloud. The same area is shown before and after damage. Most of the particles shown in the undamaged picture are probably dirt, since they do not all appear in the later photograph. The twin bands, cross twins, and hexagonal pits, however, appear only in the latter. The basal plane surface shown was obtained by cleavage of the zinc crystal at low temperature. The cleavage was done by allowing the zinc to come to temperature equilibrium in liquid nitrogen and then hitting it with a needle point.

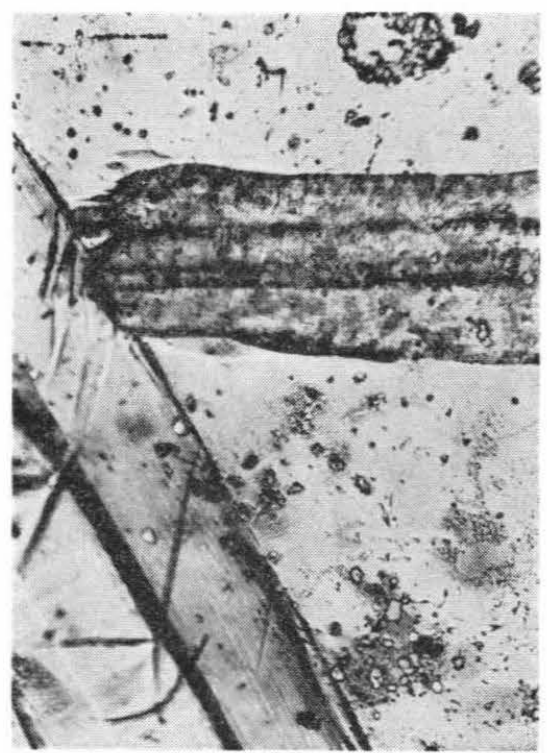

FIG. 13. Annealed surface of a zinc monocrystal cleaved along a basal plane - note appearance of many new twin bands, cross twins, and hexagonal pits-250X, 15 sec exposure. 
Damage to stellite, a polycrystalline material relatively resistant to cavitation, is shown in Fig. 14. A two-hour exposure to the large cloud produced negligible change, but the concentrated type produced the result

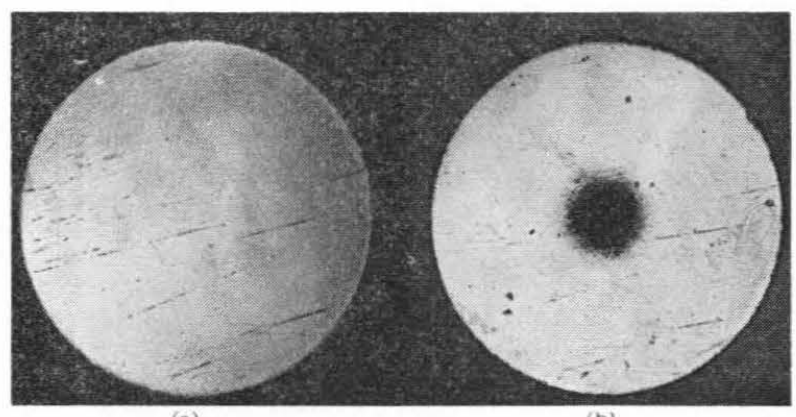

FIg. 14. Stellite specimen-magnification $40 \times$ :

(a) undamaged, (b) damaged $1 \mathrm{hr}$.

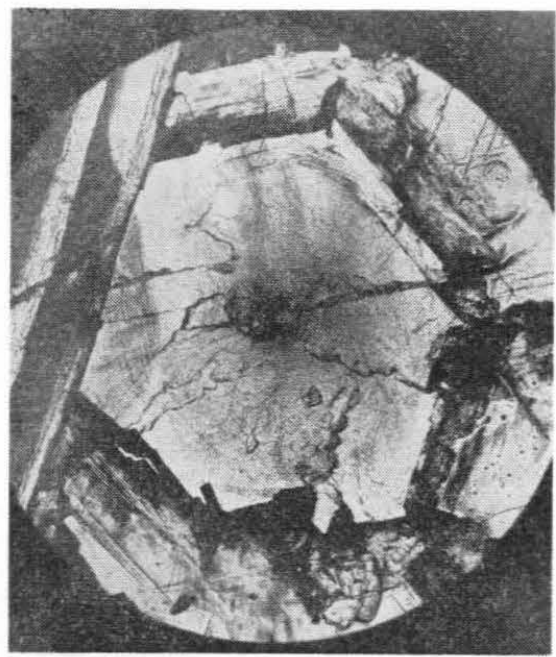

FIG. 15. Center of damaged area on (0001) plane of a single zinc crystal after 10 min exposure to $\frac{1}{16}$-in. diam cavitation bubble cloud-magnification $38 \times$. shown. Figure 15 illustrates the effect of the concentrated type of damage on a zinc monocrystal. A depression is formed in the center surrounded by a large hexagonal crack. The surface exposed to damage is the basal plane and the hexagonal cracks correspond to the hexagonal structure of the crystal. It is likely that further work with monocrystals will reveal valuable evidence concerning the damage mechanism.

Changes in structure can also be observed, even in a polycrystalline material, by the use of $x$-rays. ${ }^{11}$ Evidence of plastic deformation or cold work may be obtained very quickly. Figure 16 shows the extremely rapid change in annealed nickel upon exposure to cavitation. This figure is a back-scattered $\mathrm{x}$-ray photograph taken with radiation from a copper target at 40 kilovolts. The blurring of the spots indicates permanent deformation of the nickel structure.

Evidence as to the rate and depth of penetration of this deformation may be obtained by the technique of electrolytic polishing. This process provides a way of removing an even layer of material without introducing extraneous mechanical forces and accompanying deformation. By alternately removing layers and taking $\mathrm{x}$-rays the depth of the damaged layer may be determined. Typical $x$-ray photographs secured in this manner are shown in Fig. 17. This series indicates the depth of damage in nickel to be greater than $7 \times 10^{-3} \mathrm{~cm}$ after 30 minutes. An important observation may also be made that all the spots so sharply defined in the undamaged $x$-ray picture are nearly obliterated after exposure to the bubble cloud. If cavitation damage were a localized phenomenon such as indicated in some theories involving minute cracks, it would be difficult to understand the total absence of sharp spots after damage. Certainly the microcrystals giving rise to spots occupy very small domains.

An experiment was also carried out with this apparatus to test the widespread concept that chemical

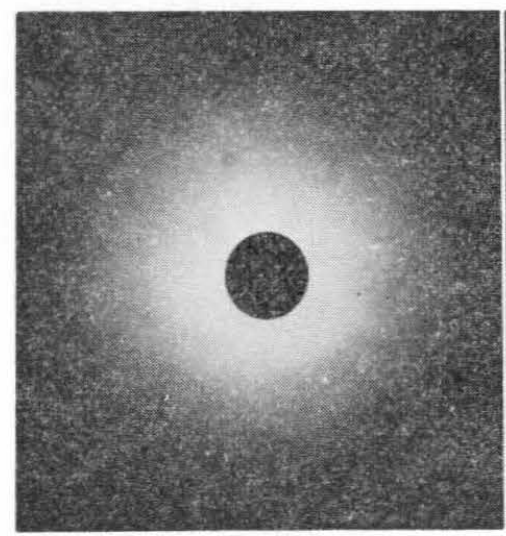

(a)

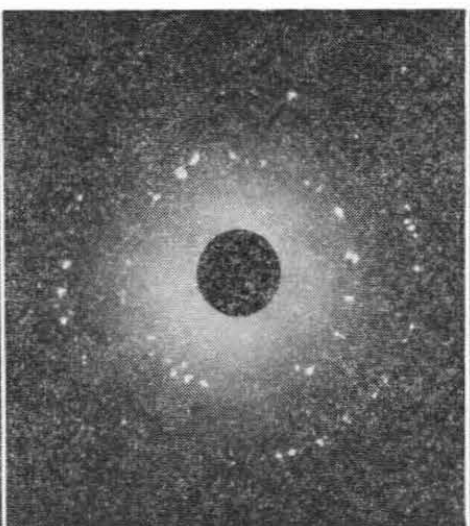

(b)

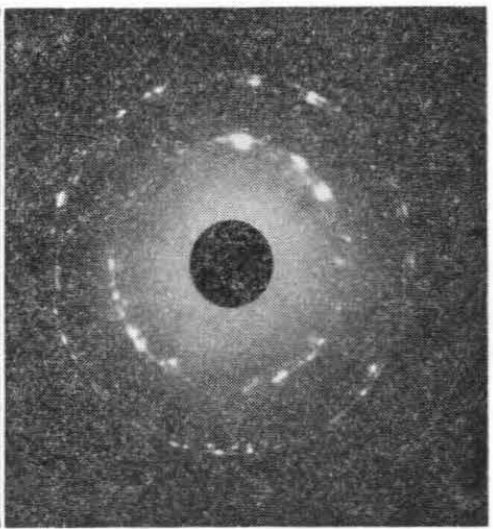

(c)

FIG. 16. X-ray diffraction pattern of nickel specimen showing rapid onset of cold work on exposure to cavitation in water : (a) before exposure, (b) after $2 \mathrm{sec}$ exposure, (c) after $10 \mathrm{sec}$ exposure.

${ }^{n}$ H. Nowotny, Werkstoffzerstörung durch Kavitation (Edwards Brothers, Inc., Ann Arbor Michigan, 1946). 


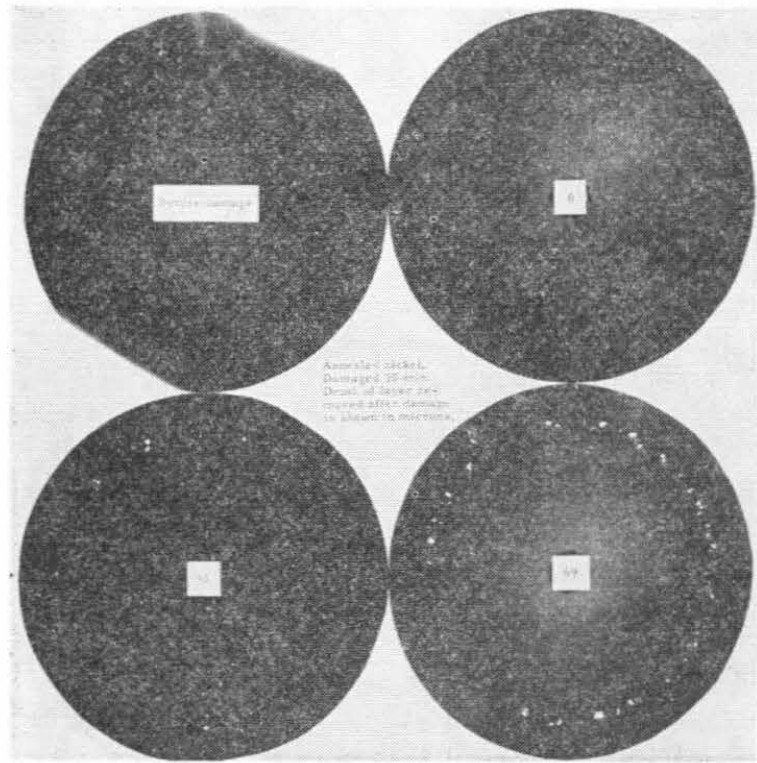

Frg. 17. Annealed nickel. Damaged $30 \mathrm{~min}$. Depth of layer removed after damage is shown in microns.

action is the primary cause of cavitation damage. A chemically inert liquid and gas were used in a damage test on nickel. Thoroughly deaerated toluene was the liquid used and helium gas was employed to maintain one atmosphere of static pressure. Figure 18 is an $\mathrm{x}$-ray pattern before and after only 2 seconds exposure, indicating the rapidity of cold working of the nickel under these conditions. Figure 19 shows the nickel surface before and after five minutes exposure to the toluene-helium cavitation. Pits in the surface before damage are due to etching. After damage, however, the surface is badly broken up, and the extent of damage appears at least as great as when water in air was used. It is therefore concluded that chemical effects are not a necessary factor in the process of cavitation damage.

Although the equipment described in this paper was not designed for specimen weight loss measurements, it was felt that some measure of material removal would facilitate correlation with existing data from testing machines of the magnetostriction type. It was found that the depth of the hole drilled in specimens formed as a 0.07 -inch diameter cylinder was a relative measure of resistance to damage. The diameter of the hole remained at about 0.015 inch and the depth was allowed to progress to about 0.005 inch in some cases. For brass, this represented a weight loss of 7 micrograms, which was too small for direct weight loss measurement. Table II shows the results for some of the materials tested.

\section{SUMMARY}

A new method for the production of accelerated cavitation damage has been developed. The apparatus is inexpensive and efficient, and the relative resistance of surfaces to damage is easily obtained. An advantage of the method is that the specimen itself is nearly stationary and hence not subject to acceleration forces. Mathematical analysis of the bubble motion should also be simplified.

Experiments have been carried out with various surfaces. Photomicrography and $\mathrm{x}$-ray techniques have

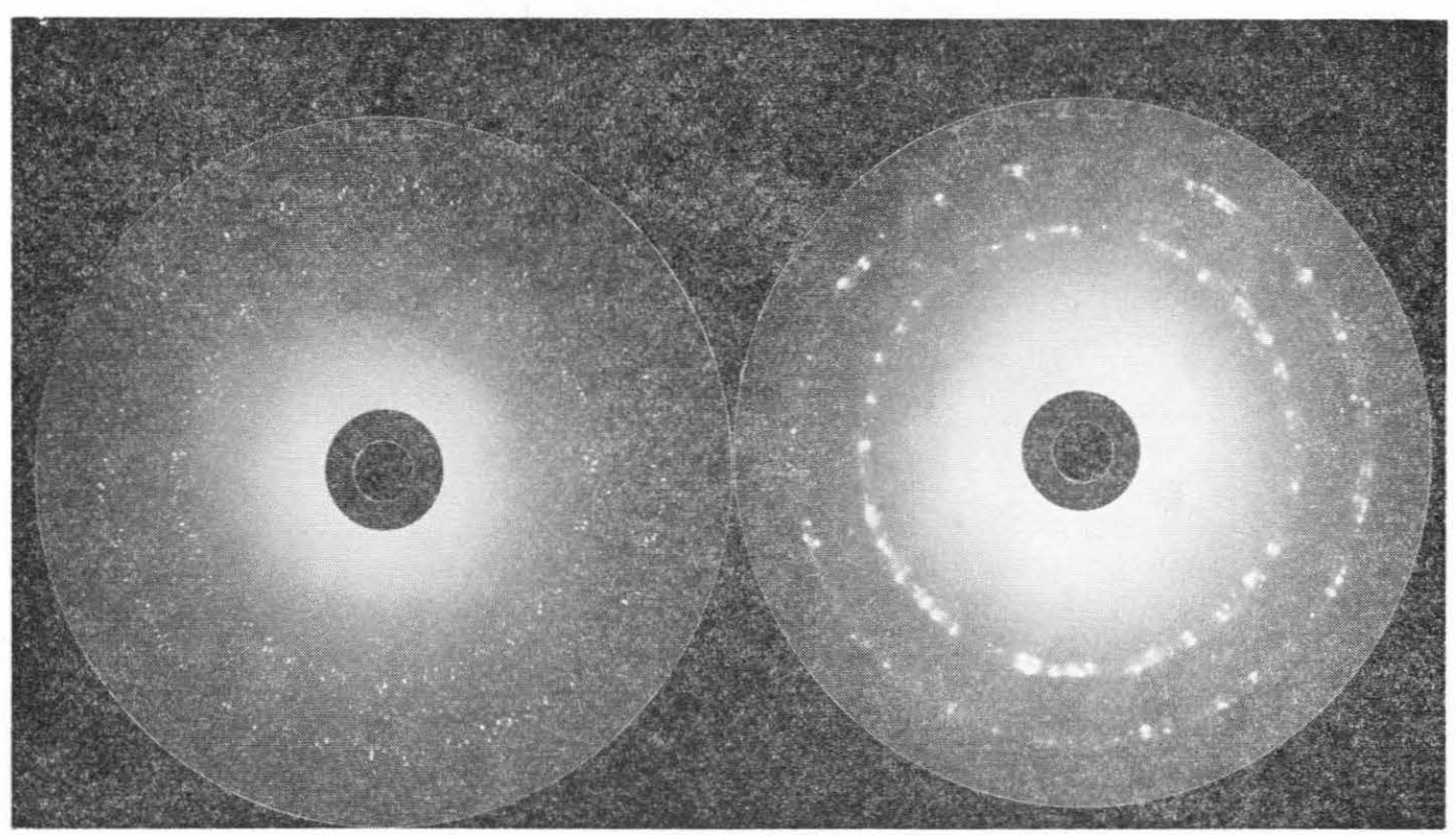

FIG. 18. X-ray diffraction pattern of nickel specimen showing rapid onset of cold work on exposure to cavitation in toluene in a helium atmosphere: (a) before exposure, (b) after $2 \mathrm{sec}$ exposure. 
TABLE II.

\begin{tabular}{|c|c|c|c|c|c|c|c|c|c|c|c|}
\hline \multirow{2}{*}{$\begin{array}{l}\text { Material (arranged in } \\
\text { order of increasing } \\
\text { hardness) }\end{array}$} & \multirow{2}{*}{$\begin{array}{l}\text { Composition } \\
\text { percent }\end{array}$} & \multirow{2}{*}{$\begin{array}{l}\text { Hardness } \\
\text { (Brinell) }\end{array}$} & \multirow{2}{*}{$\begin{array}{l}\text { Ultimate } \\
\text { tensile } \\
\text { strength } \\
\left(\mathrm{psi} \times 10^{3}\right)\end{array}$} & \multirow{2}{*}{$\begin{array}{c}\text { Modulus } \\
\text { of } \\
\text { elasticity } \\
\text { (psi } \\
\left.\times 10^{6}\right)\end{array}$} & \multicolumn{7}{|c|}{ Depth of cavitation damage hole in microns $\left(10^{-4} \mathrm{~cm}\right)$} \\
\hline & & & & & $10 \mathrm{sec}$ & $1 \mathrm{~min}$ & $15 \mathrm{~min}$ & $30 \mathrm{~min}$ & $1 \mathrm{hr}$ & $2 \mathrm{hr}$ & $3 \mathrm{hr}$ \\
\hline Aluminum (soft) & & 16 & 16 & 10 & 10 & 80 & & & & & \\
\hline Titanium (annealed) & & 58 & 79 & 16 & & & 43 & 78 & & & \\
\hline Nickel & & 90 & 50 & 30 & & & 80 & 115 & & & \\
\hline Molybdenum & & 120 & 57 & 50 & & & & 10 & 25 & 60 & 100 \\
\hline Brass & $\mathrm{Cu} 70, \mathrm{Zn} 30$ & 123 & 56 & 13 & & 1 & 85 & 128 & & & \\
\hline Stainless steel & Cr $18, \mathrm{Ni} 8$ & 163 & 102 & 29 & & & 15 & 28 & & & \\
\hline Titanium 75-A & & 203 & 80 & 16 & & & & & 30 & 66 & \\
\hline Steel $(4130)$ & & 258 & 130 & 30 & & & & & 32 & 55 & \\
\hline Tungsten & & 350 & 597 & 51 & & & & 0 & 0 & 3 & 12 \\
\hline Titanium 130-A & Ti 92, Mn 7.9 & 351 & 130 & 16 & & & & 0 & 3 & 16 & \\
\hline Colmonoy & & 400 & 61 & & & & & 0 & 3 & 18 & 34 \\
\hline Titanium $15 \mathrm{C}-\mathrm{A}$ & $\begin{array}{l}\text { Ti } 96, \text { Cr } 2.7 \\
\text { Fe } 1.3\end{array}$ & 437 & 150 & 16 & & & & 0 & 0 & 3 & 26 \\
\hline Stellite & $\begin{array}{l}\text { Co } 55, \text { Cr 33, } \\
\text { W } 6\end{array}$ & 495 & 100 & 36 & & & & 0 & 3 & 14 & 29 \\
\hline Pyrex & & Moh 5 & & 10 & & & 120 & & & & \\
\hline Fused quartz & & Moh 7 & & 9 & & & 100 & & & & \\
\hline
\end{tabular}

yielded metallurgical information of value in determining physical properties for cavitation resistant materials. Such information indicates that stresses due to cavitation must be at least of the order of 50000 psi. It also indicates an interaction of many of the properties of the solid, so that consideration of any single property would be misleading. It appears that a material exposed to cavitation sooner or later under-

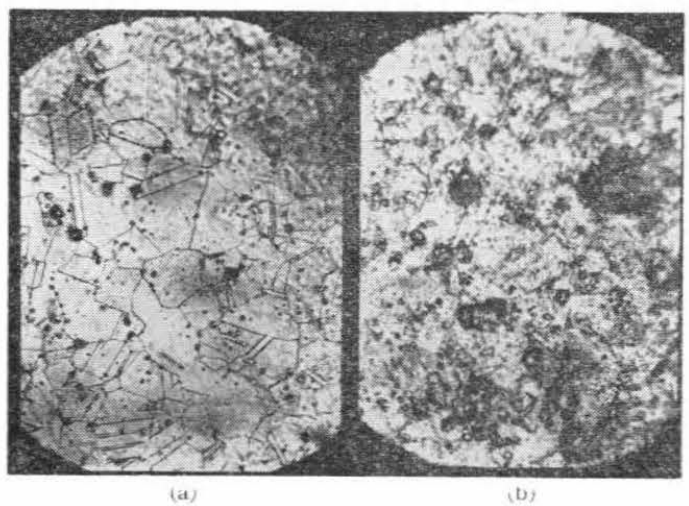

FIG. 19. Annealed and etched surface of nickel specimen exposed to cavitation in liquid toluene in a helium atmosphere: (a) before exposure, (b) after 5 min exposure. goes plastic deformation. This plastic deformation is a cold-working process which leads to fatigue and failure of the specimen.

Experiments using inert materials show essentially the same damage effects. It is to be concluded that chemical effects are not of primary importance. This does not mean that they do not contribute at all, for it is well known that chemical reactions can affect the fatigue properties of some substances.

\section{ACKNOWLEDGMENTS}

This study was supported by the Office of Naval Research, Mechanics Branch. The author wishes to thank Professor M. S. Plesset for both suggesting the problem and for active participation throughout the entire study.

Professor Pol Duwez, Professor David Wood, and Professor Thad Vreeland, Jr., of the Engineering Division of the California Institute of Technology, contributed greatly in the metallurgical aspects of the work.

Especial thanks is due Dr. Leon Camp of the Bendix Aviation Corporation both for helpful discussion and for supplying the ceramic transducers used in this project. 